Conf-940592--14

UCRL-JC-117006

PREPRINT

\title{
Theory of Recombination X-Ray Lasers Based on Optical-Field Ionization
}

\author{
D. C. Eder \\ P. Amendt \\ L. B. DaSilva \\ T. D. Donnelly \\ R. W. Falcone \\ R. A. London \\ M. D. Rosen \\ S. C. Wilks
}

This paper was prepared for submittal to the 4th International Colloquium on X-Ray Lasers

Williamsburg, VA

May 16-20 1994

\section{December 1994}

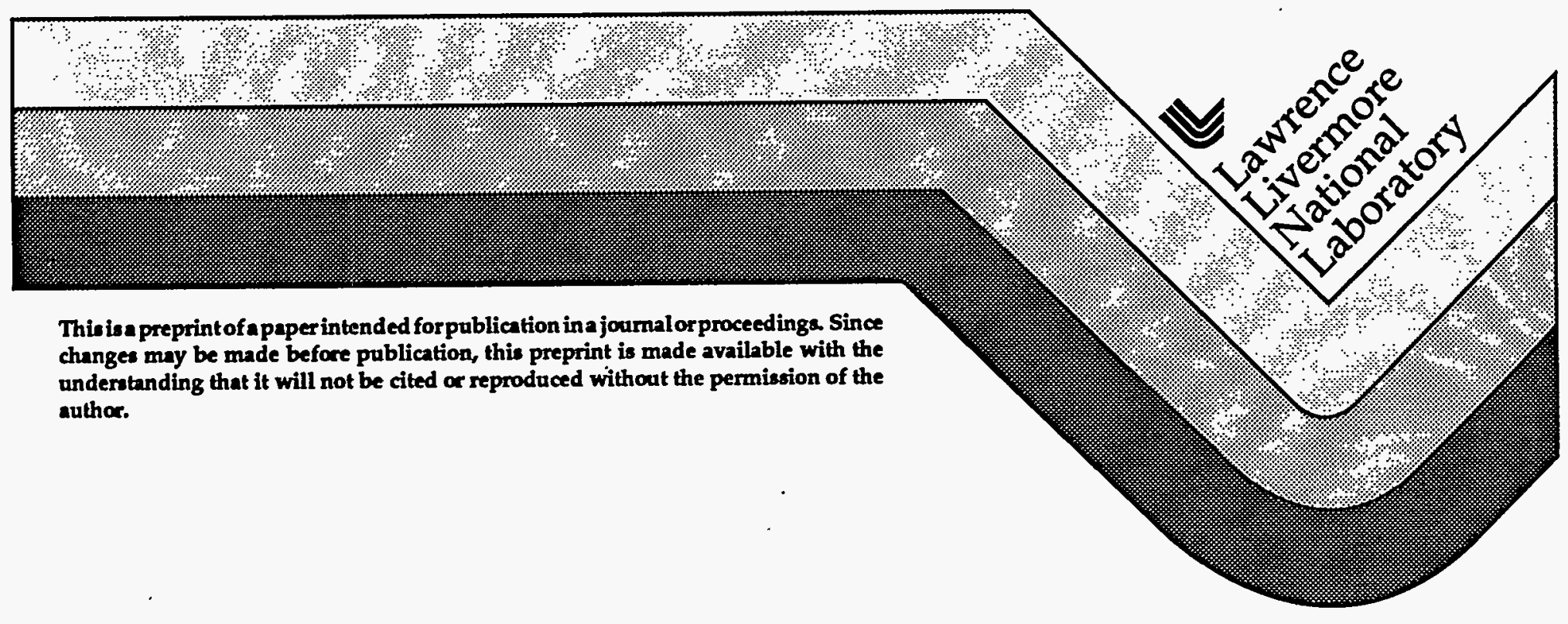

This is a preprint of a paper intended for publication in a journal or proceedinga. Since changes may be made before publication, this preprint is made available with the understanding that it will not be cited or reproduced without the permission of the author. 


\section{DISCLAIMER}

This document was prepared as an account of work sponsored by an agency of the United States Government. Neither the United States Government nor the University of California nor any of their employees, makes any warranty, express or implied, or assumes any legal liability or responsibility for the accuracy, completeness, or usefulness of any information, apparatus, product, or process disclosed, or represents that its use would not infringe privately owned rights. Reference herein to any specific commercial product, process, or service by trade name, trademark, manufacturer, or otherwise, does not necessarily constitute or imply its endorsement, recommendation, or favoring by the United States Government or the University of California. The views and opinions of authors expressed herein do not necessarily state or reflect those of the United States Government or the University of California, and shall not be used for advertising or product endorsement purposes. 


\section{DISCLAIMER}

Portions of this document may be illegible in electronic image products. Images are produced from the best available original document. 


\title{
Theory of Recombination X-Ray Lasers Based on Optical-Field Ionization
}

\author{
D. C. Eder*, P. Amendt*, L. B. DaSilva*, T. D. Donnelly ${ }^{\dagger}$, \\ R. W. Falcone ${ }^{\dagger}$, R. A. London*, M. D. Rosen*, and S. C. Wilks* \\ * Lawrence Livermore National Laboratory, Livermore, CA 94550 \\ †University of California at Berkeley, Berkeley, CA 94720
}

\begin{abstract}
Ultrashort-pulse, high-intensity laser drivers have the potential for creating tabletop-size $\mathrm{x}$-ray lasers by ionizing the target gas via the electric field of the laser pulse. For appropriate plasma conditions following ionization, lasing can occur during the subsequent rapid recombination. A review of the theory and modeling for these opticalfield-ionized $x$-ray lasers is presented. Particular attention is given to the issues of electron heating and ionization-induced refraction. We summarize modeling in support of experiments where evidence of lasing in H-like Li at $135 \AA$ was obtained. In addition, we present modeling results for lasing in Li-like $\mathrm{N}$ at $247 \AA$. We briefly discuss new applications appropriate for tabletop-size high-repetition-rate $\mathrm{x}$-ray lasers.
\end{abstract}

\section{INTRODUCTION}

Since the last conference in this series in Schliersee, Germany, ${ }^{1}$ the major development in recombination x-ray lasers based on optical-field ionization has been the observation of faster than linear growth of the intensity with length for the $\mathrm{L}_{\alpha}$ line in $\mathrm{H}$-like $\mathrm{Li}$ at $135 \AA$. The initial observations ${ }^{2}$ were at the RIKEN laboratory in Japan with similar results being obtained in a UC Berkeley/LLNL collaboration. ${ }^{3}$ Other observations that are indicative of lasing in $\mathrm{H}$-like $\mathrm{Li}$ are the directionality measured at RIKEN and the short period of emission $(\Delta t \approx 20 \mathrm{psec})$ seen first in the UC Berkeley/LLNL experiment and subsequently also observed in the RIKEN experiment. ${ }^{4}$ In addition to the experiments in $\mathrm{Li}$, there was an observation of enhanced intensity for a transition between excited states in Li-like $\mathrm{N}$ (5f-3d line at $512 \AA) .5$ However, for all these experiments the measured gainlength product is relatively small $(g L \leq 4)$. While lasing has most likely been observed, a definitive demonstration and sufficient output for applications awaits experiments with significantly larger gain-length products. In the RIKEN experiments, the lasing length is limited by the power available from their field ionizing laser. ${ }^{4}$ Ionization-induced refraction appears to be the factor limiting the length in the Berkeley/LLNL experiments. ${ }^{3}$ Recent experiments showing plasma waveguiding are very relevant to solving the refraction problem. 6,7

There has also been significant progress in the theory and modeling of recombination $\mathrm{x}$-ray lasers based on optical-field ionization. The importance of collisional heating at higher densities has been calculated by a number of authors, $8-9$ but there are still some unresolved issues that would benefit from additional theory and experiments. There has been significant progress in understanding the 
importance of ionization-induced refraction. $10-12$ While using an additional laser to create a plasma waveguide is one potential solution to the problem of refraction, 6,7 we are also investigating optimum placement of the gas jet or ablating vapor with respect to the location of vacuum best focus and the relative benefit of larger focal spots that give shallower density gradients as compared to using higher intensities that give narrow but nearly flat-top density profiles. Recent modeling for $\mathrm{H}$-like $\mathrm{Li}$ shows the importance of recombination and collisional cascade heating and has shown the effects of two temperature electron distributions on the calculated gain coefficient. ${ }^{13}$ Modeling for Li-like $\mathrm{N}$ has shown that a significant gain-length product is possible by using an element with lower ionization potential which allows for a larger spot size. ${ }^{14}$

Lasing during rapid recombination following field ionization is only one of the approaches being considered for tabletop-size x-ray lasing. A number of different tabletop-size $x$-ray lasers, all with relatively small gain-length products, have been demonstrated in the past few years. 15 In this proceedings Rocca, etal., present the first demonstration of a tabletop system based on a capillary discharge that achieved a gain-length product of 7 in Ne-like $\mathrm{Ar}$ at $469 \AA .{ }^{16} \mathrm{In}$ addition, a variation of the OFI $x$-ray scheme, discussed in this paper, using circularly polarized laser was recently used to achieve a gain-length product of 11 in Pd-like Xe at $418 \AA .17$ The basic difference in this latter scheme, as compared to the one discussed here, is that the electrons ionized by the circularly polarized field are left with enough energy to collisional populate the upper-laser state as compared to having cold electrons that populate an upper-laser state via rapid recombination. Since both schemes are based on forming a plasma using a confocal geometry, problems associated with ionization-induced refraction that affect the scheme discussed in this paper will also be important for the other OFI approach for shorter wavelengths where higher densities are required.

Following an overview of theoretical issues for OFI x-ray lasing discussed in Sec. II, we summarize modeling results for $\mathrm{H}$-like $\mathrm{Li}$ and Li-like $\mathrm{N}$ in Secs. III and IV. In Sec. V, we briefly discuss some appropriate applications for table-top size x-ray lasers having relatively high repetition rates. We conclude with some comments on future prospects in Sec. VI.

\section{THEORETICAL ISSUES FOR OFI X-RAY LASING}

During rapid recombination following field ionization, lasing can occur between two excited states and between an excited state and the ground state. Most of the attention has been on lasing down to the ground state because of the relatively shorter wavelengths that can be achieved. For applications requiring less energetic photons, lasing between excited states is a potential x-ray source. There are some initial results for transitions between excited states of Li-like $\mathrm{N}$, e.g., $5 \mathrm{f}-3 \mathrm{~d}$ line at $512 \AA .5$ In the rest of this paper we will restrict our attention to lasing to the ground state, although many of the results can be applied to lasing between excited states.

The basic requirement for achieving a significant gain coefficient in lasing down to the ground state, e.g., $n=2$ to $n=1$ or the $\mathrm{L}_{\alpha}$ transition in $\mathrm{H}$-like ions, is that the recombination to the upper Rydberg levels and subsequent collisional cascade down 
to the upper-laser level occur on a faster time scale than the radiative and collisional filling of the lower-laser (ground) level. This requirement places constraints on the density and temperature following field ionization. A lower density requires a lower temperature to ensure sufficiently fast 3-body collisional recombination. Except at very high intensities where Raman heating can be important, 18 the dominate heating mechanisms are above-threshold-ionization (ATI) and electron collisions. ATI heating is associated with the phase mismatch between the time of ionization and the peak of the oscillatory electric field. 19,20 Since the amount of energy given to the electron for a given phase mismatch depends on the quiver velocity, there is a benefit in using a shorter wavelength ionizing laser. Collisions by the rapidly oscillating electrons can give significant heating at higher densities. $8,9,20$ There are differences in the choice of the Coulomb logarithm used in the different calculations that result in significant differences in the predicted heating. ${ }^{14}$ For the $\mathrm{H}$-like $\mathrm{Li}$ experiments, the densities are low enough that collisional heating is not be important for any of the choices used for the Coulomb logarithm. For the proposed Li-like $\mathrm{N}$ scheme discussed in Sec. IV., the collisional heating contribution varies from being very minor to being very significant depending on the model. Additional theoretical work is required to accurately treat collisional heating for OFI plasma parameters.

In most conventional $x$-ray lasing schemes a plasma is heated with a laser using a line focus geometry and the $x$-ray lasing axis is perpendicular to the driving laser axis. However, in OFI lasing schemes a confocal geometry is used and refraction of the driving laser as a result of field ionization is a major issue. In general, laser pulses have maximum intensity on axis which results in the electron density also having a maximum on axis giving the potential for refraction. One approach to reducing refraction is to use a short wavelength driving laser and operate at a low density. This is the case for the RIKEN experiments where $0.25 \mu \mathrm{m}$ light is used and the electron density was measured to be of order $10^{17} \mathrm{~cm}^{-3} .^{2}$ (As discussed below, modeling results for $\mathrm{H}$-like $\mathrm{Li}$ predict very little gain for this low value of density.) Even if the density electron density is a factor of ten higher, refraction should not be a major problem. The UC Berkeley/LLNL experiments use a longer wavelength of $0.4 \mu \mathrm{m}$, and a simple calculation shows that the fall off in gain for lengths longer than $1.5 \mathrm{~mm}$ could be the result of refraction. ${ }^{3}$ While there has been significant progress in modeling refraction, $10-12$ a detailed study that selfconsistently calculates transverse intensity and electron density profiles has not been completed. We have started such a study with one issue being the optimum placement of the gas jet or ablating vapor with respect to the location of vacuum best focus. If more energy is available by using an element with a lower ionization potential or by increasing the driving laser energy, there is a question if it is better to increase the size of the focal spot and thus produce a shallower density gradient or to use higher intensity to give a narrow but nearly flat-top density profile. We take the first approach in our discussion of potential lasing in Li-like N in Sec. IV. For some situations, the second approach might be better. We believe that the refraction study in progress will help answer these questions. 


\section{SUMMARY OF MODELING FOR H-LIKE LI AT $135 \AA$}

Motivated by the recent experiments in $\mathrm{H}$-like $\mathrm{Li}$, there has been interesting modeling that has led to an unexplained discrepancy with experimental measurements of density. The electron density measured in the RIKEN experiment of order $10^{17} \mathrm{~cm}^{-3}$ is too low to produce any significant gain for an arbitrary cold electron distribution. ${ }^{13}$ (Our understanding of the H-like $\mathrm{Li}$ experiments is based on the assumption of a two-component electron distribution, with the colder component arising from the outer electron and the hotter component arising from field ionization of the two inner electrons.) The key factor that has to be included in the modeling, to show the discrepancy with the density measurement, is heating of the free electrons by collisions associated with 3-body recombination and cascade to the lower levels. While a constant temperature of a fraction of $1 \mathrm{eV}$ could produce significant gain for an electron density of $10^{17} \mathrm{~cm}^{-3}$, the free electrons rapidly heat to temperatures above $1 \mathrm{eV}$ thus slowing down recombination and inhibiting gain. Since the density measurement is based on time integrated data of He-like lines, there is a potential that the density could be higher for some time in a region that could have significant gain. Additional data on densities would be very useful.

The recent modeling of $\mathrm{H}$-like $\mathrm{Li}$ also addressed the question of the effect of two electron components with different temperatures on the calculated gain coefficient. ${ }^{13}$ Our initial calculations neglected the effect of the hotter distribution. ${ }^{3}$ The modeling shows that the hotter component has the largest reduction on the gain when its temperature is of order $100 \mathrm{eV}$ for a cold component having a temperature of $1 \mathrm{eV}$. For very high temperatures, the hot component no longer slows down the recombination and cascade because collisions with the hot component are very infrequent. However, an interesting effect results from having the same number of recombining cold electrons as ions. The electrons rapidly recombine to the upper Rydberg levels but this results in a large reduction in the free electron density which slows down the collisional cascade to the lower levels. In this case, one is better off having a "hot" component having a temperature of order $10 \mathrm{eV}$ or less than having a hot component that does not interact at all because the temperature is too high. For details of these calculations see the proceedings in this collection by Donnelly. ${ }^{13}$

\section{SUMMARY OF MODELING FOR LI-LIKE N at $247 \AA$}

One solution for problems associated with refraction is to use an element with a lower ionization potential which thus requires a lower laser intensity to field ionize. This allows for the use of a larger focal spot which gives shallower density gradients and less refraction. For this reason we have done calculations for lasing in Li-like $\mathrm{N}$ at $247 \AA .14$ The required intensity to ionize $\mathrm{N}$ to the required He-like ionization stage is $3 \times 10^{16} \mathrm{Wcm}^{-2}$. For a pulse duration of $100 \mathrm{fsec}$ and an energy of $0.5 \mathrm{~J}$, a focal spot with a radius of $70 \mu \mathrm{m}$ is possible. A very rough estimate of the lasing length as a result of refraction is given by $L=2 b\left[n_{\mathrm{d}}\left(n_{\mathrm{e}} \ln 2\right)\right]^{1 / 2}$, where $n_{\mathrm{c}}$ is the critical density, $n_{\mathrm{e}}$ is the electron density, and $b$ is the radius of the focal spot. ${ }^{3}$ For a wavelength of $0.4 \mu \mathrm{m}$, an electron density of $2.5 \times 10^{19} \mathrm{~cm}^{-3}$, and a focal spot radius of $70 \mu \mathrm{m}$, a lasing length of $3 \mathrm{~mm}$ is estimated. The density assumed here results in this length being only a factor of 2 greater than the $1.5 \mathrm{~mm}$ 
length in the UC Berkeley/LLNL experiments where refraction appears to be limiting the length. The need for the higher density is because a cold electron component, with a temperature significantly below that obtained from ATI heating, can not be justified in this case. To obtain a significant gain-length product $(g L>5)$ with only a lasing length of $3 \mathrm{~mm}$, one requires a gain coefficient greater than $15 \mathrm{~cm}^{-1}$. Based on our calculations of gain this implies that the temperature must be $12 \mathrm{eV}$ or less for an electron density of $2.5 \times 10^{19} \mathrm{~cm}^{-3}$ as shown in Fig. 1. Our estimates of ATI and collisional heating indicate that a temperature as low as $10 \mathrm{eV}$ are possible for this system. 14

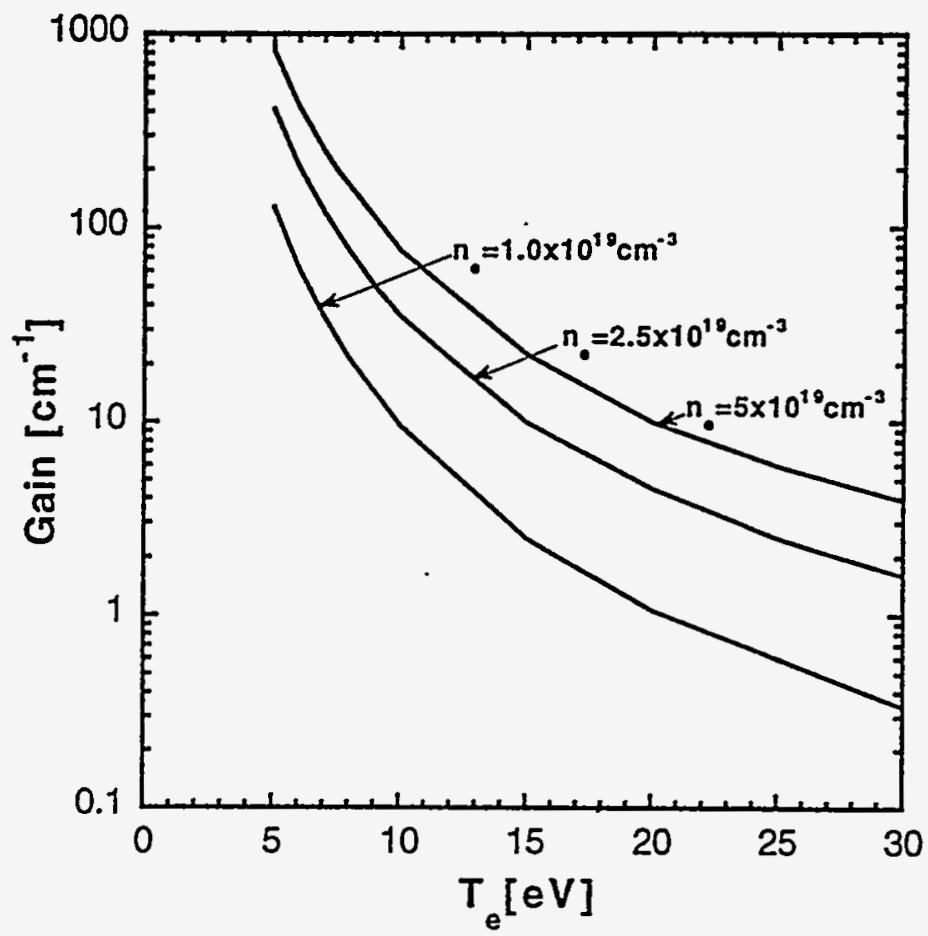

FIGURE 1. Calculations of the gain for the $3 d_{5 / 2}$ to $2 p_{3 / 2}$ transition in Li-like $N$ at $247 \AA$.

\section{APPLICATIONS FOR TABLETOP-SIZE X-RAY LASERS}

An important aspect of most existing and proposed tabletop-size $\mathrm{x}$-ray lasers is a relatively high repetition rate. The current repetition rate for the OFI Pd-like Xe Xray laser operating at $418 \AA$ is $10 \mathrm{~Hz}, 17$ and the laser used to pump the OFI H-like Li scheme at $135 \AA$ could also have this repetition rate. This allows one to have quite high average powers, with relatively small amount of energy per pulse, that can exceed the average power available from current 3rd generation synchrotron facilities. For example, the Advance Light Source (ALS) synchrotron facility is quoted to have an average flux of $2 \times 10^{13}$ photons/sec at $150 \AA$ for a $10^{-4}$ bandwidth. ${ }^{21}$ An $x$-ray laser operating at $10 \mathrm{~Hz}$ would require $25 \mu \mathrm{J}$ per pulse to have the same average power. Most tabletop-size $\mathrm{x}$-ray laser schemes are calculated to have a saturated energy per pulse of this order or greater. The big advantage of tabletop x-ray lasers is that they would be low cost and small enough to be in individual laboratories. A large number of the applications currently being studied 
using synchrotrons are not well suited to tabletop $x$-ray lasers because of the low bandwidth required (synchrotron flux levels are much higher for a $10^{-2}$ bandwidth) or the desire to have continuous tunability. However, there are many applications currently being studied with synchrotrons and other devices, as well as applications that can not be studied with synchrotrons, that are very well matched to the output properties of tabletop $\mathrm{x}$-ray lasers. ${ }^{22}$

Photoelectron spectroscopy of core electrons to study dynamical processes on surfaces is one example where tabletop $x$-ray lasers could make an important contribution. Continuous tunability is not required because the measurement of the energy of the ejected electron combined with a well know photon energy (i.e., the narrow bandwidth $x$-ray laser source) determines the time dependence of the binding energy of the core level. The shifts in the binding energy of the core levels give important information on the dynamics of impurities, chemical processes, etc. In many case these shifts are relatively small which places the requirement on having a narrow bandwidth source. The quasi-continuous output of synchrotrons (tens of psec pulses separated by time intervals in the nsec range) makes the study of dynamical processes and the use of pump/probe techniques very difficult. In photoelectron spectroscopy a very wide range of photons wavelengths are of interest including the relatively long wavelengths $(\lambda>400 \AA)$ of current tabletop $x$ ray lasers that have obtained significant gain-length products.

\section{CONCLUSIONS AND FUTURE PROSPECTS}

Experiments in $\mathrm{H}$-like $\mathrm{Li}$ at $135 \AA$ have provided evidence that is very indicative of lasing during rapid recombination following optical-field ionization. In addition, substantial progress has been made in the theory and modeling of this new type of $\mathrm{x}$-ray laser. Continued work in electron heating and refraction is clearly desired. Modeling has shown that significant gains are possible for the H-like Li scheme provide there is a cold electron component and the density is approximately $10^{18} \mathrm{~cm}^{-3}$. There is a conflict between modeling and current experiments in that the measured density in the RIKEN experiment is significantly.less than $10^{18} \mathrm{~cm}^{-3}$. Additional measurements of density are clearly needed. The modeling results for $\mathrm{Li}$ like $N$ at $247 \AA$ show that a significant gain-length product $(g L>5)$ is possible provided the electron temperature is of order $10 \mathrm{eV}$ for electron densities of order $10^{19} \mathrm{~cm}^{-3}$.

The future prospects for tabletop x-ray lasers, in general, are very good with many applications to explore. The outlook for $\mathrm{x}$-ray lasers based on rapid recombination following field ionization appears to be very promising provided that work continues on propagation problems. Modeling for these schemes would benefit greatly from more measurements of electron density and temperature. Hopefully by the time of the next conference in this series these goals will be met.

\section{Acknowledgments}

Work performed under the auspices of the U. S. Department of Energy by Lawrence Livermore National Laboratory under Contract W-7405-ENG-48. 


\section{References}

1. X-Ray Lasers 1992, Proceedings of the 3rd International Colloquium on X-Ray Lasers, IOP Conference Series 125, edited by E. E. Fill (Institute of Physics, Bristol, England, 1992).

2. Y. Nagata, K. Midorikawa, M. Obara, H. Tashiro, and K. Toyoda, Phys. Rev. Lett. 71, 3774 (1993).

3. D. C. Eder, P. Amendt, L. B. DaSilva, R. A. London, B. J. MacGowan, D. L. Matthews, B. M. Penetrante, M. D. Rosen, S. C. Wilks, T. D. Donnelly, R. W. Falcone, and G. L. Strobel, Phys. Plasmas 1, 1744 (1994).

4. K. Midorikawa, Y. Nagata, M. Obara, H. Tashiro, and K. Toyoda, these proceedings.

5. S. Borgström, E. Fịll, J. Larsson, T. Starczewski, S. Svanberg, and C.-G. Wahiström, these proceedings.

6. C. G. Durfee III and H. M. Milchberg, Phys. Rev. Lett. 71, 2409 (1993).

7. H. M. Milchberg, C. G. Durfeee III, and J. Lynch, these proceedings.

8. S. C. Rae and K. Burnett, Phys. Rev. A 46, 2077 (1992).

9. P. Pulsifer, J. P. Apruzese, J. Davis, and P. Kepple, Phys. Rev. A 49, 3958 (1994).

10. W. P. Leemans, C. E. Clayton, W. B. Mori, K. A. Marsh, P. K. Kaw, A. Dyson, and C. Joshi, Phys. Rev. A 462077 (1992).

11. S. C. Rae, Opt. Commun. 97, 25 (1993).

12. X. Liu and D. Umstadter, OSA Proceedings on Shortwavelength V: Physics with Intense Laser Pulses, edited by M. D. Perry and P. B. Corkum (Optical Society of America, Washington, DC, 1993), Vol. 17, p. 45.

13. T. D. Donnelly, T. E. Glover, M. Hofer, E. A. Lipman, R. W. Lee, L. Da SIlva, D. C. Eder, S. Mrowka, and R. W. Falcone, these proceedings.

14. P. Amendt, D. C. Eder, R. A. London, B. M. Penetrante, and M. D. Rosen, SPIE Proceedings, Conference on Short-Pülse High-Intensity Lasers and Applications, Edited by H. A. Baldis (SPIE, Bellingham. WA, 1993), Vol. 1860, p. 140.

15. See reference 1, SPIE Proceedings, Ultrashort-Wavelength Lasers II. edited by S. Suckewer (SPIE, Bellingham, WA, 1994), Vol. 2012, or these proceedings.

16. J. J. Rocca, F. G. Tomasel, V. N. Shlyaptsev, O. D. Cortazar, J. L. A. Chilla, and G. Giudice, these proceedings and J. J. Rocca, V. Shlyaptsev, F. G. Tomasel, O. D. Cortazar, D. Hartshorn, and J. L. A. Chilla, Phys. Rev. Lett. 73, 2192 (1994).

17. B. E. Lemoff, G. Y. Yin, C. L. Gordon, III, C. P. J. Barty, and S. E. Harris, submitted to Phys. Rev. Lett.

18. D. C. Eder, P. Amendt, and S. C. Wilks, Phys. Rev. A 45, 6761 (1992).

19. P. B. Corkum, N. H. Burnett, and F. Brunel, Phys. Rev. Lett. 62, 1259 (1989).

20. B. M. Penetrante and J. N. Bardsley, Phys. Rev. A 43, 3100 (1991).

21. ALS Beamlines Lawrence Berkeley Laboratory PUB-3104 (1992).

22. D. C. Eder, P. Amendt, C. B. Dane, L. B. DaSilva, L. A. Hackel, M. R. Hermann, R. A. London, B. J. MacGowan, D. L. Matthews, M. D. Rosen, and S. C. Wilks, Proceedings of the International Conference on LASERS '92, edited by C. P. Wang ( Press, McLean, VA, 1993), p. 67. 